\title{
The Precaution Adoption Process Model in Describing Emergency Action Plan Adoption
}

\author{
Samantha E. Scarneo-Miller \\ West Virginia University, samantha.scarneomiller@hsc.wvu.edu \\ Douglas J. Casa \\ University of Connecticut, douglas.casa@uconn.edu \\ Shuang Yin \\ University of Connecticut, shuang.yin@uconn.edu \\ Timothy E. Moore \\ University of Connecticut, timothy.e.moore@uconn.edu \\ Yuki Murata \\ Nagoya University, yuki.murata05@gmail.com \\ See next page for additional authors
}

Follow this and additional works at: https://nsuworks.nova.edu/ijahsp

Part of the Health and Medical Administration Commons, Health Services Administration Commons, Sports Sciences Commons, and the Translational Medical Research Commons

\section{Recommended Citation}

Scarneo-Miller SE, Casa DJ, Yin S, Moore TE, Murata Y, Register-Mihalik JK, et al. The Precaution Adoption Process Model in Describing Emergency Action Plan Adoption. The Internet Journal of Allied Health Sciences and Practice. 2021 Jan 01;19(2), Article 2.

This Manuscript is brought to you for free and open access by the College of Health Care Sciences at NSUWorks. It has been accepted for inclusion in Internet Journal of Allied Health Sciences and Practice by an authorized editor of NSUWorks. For more information, please contact nsuworks@nova.edu. 


\title{
The Precaution Adoption Process Model in Describing Emergency Action Plan Adoption
}

\begin{abstract}
Purpose: The purpose of this study was to identify the ability of the Precaution Adoption Process Model (PAPM) to classify athletic trainers (ATs) and athletic directors (ADs) readiness to act with regards to adopting an emergency action plan (EAP), and describe factors influencing PAPM stages. Method: We used a cross-sectional questionnaire design to evaluate readiness to act for developing an EAP among ATs and ADs working in United States high schools. The PAPM is a participant-driven model to identify someone's readiness to act or change and has seven stages: Unaware, Unengaged, Undecided, Decided not to Act, Decided to Act, Acting and Maintaining. Prevalence ratios (PRs) with 95\% confidence intervals $(95 \% \mathrm{Cl})$ were calculated. Results: A majority of ATs and ADs reported maintaining an EAP in their secondary school (AT "Maintaining" $=84.4 \%$, AD "Maintaining" $=68.7 \%$ ). ADs were more likely to be staged as "Decided not to Act" (PR=0.14, 95\% $\mathrm{Cl}=0.05,0.41)$ for the development of an EAP compared to ATs. ATs were more likely to be staged as "Maintaining" ( $P R=1.23,95 \% \mathrm{Cl}=1.16,1.30)$ for the development of an EAP compared to ADs. Conclusions: The PAPM appears to be able to classify AT and AD readiness to act with adoption of an EAP. However, statistical modeling struggles to identify predictors for the various stages. Future research should aim to use the PAPM stages in the development of tailored interventions.
\end{abstract}

\section{Author Bio(s)}

Samantha E. Scarneo-Miller, $\mathrm{PhD}$, ATC, is the program director of the athletic training program and assistant professor in the Division of Athletic Training in the School of Medicine at West Virginia University. She is also a licensed athletic trainer.

Douglas J. Casa, PhD, ATC, is the Chief executive officer for the Korey Stringer Institute and Professor in the Department of Kinesiology at the University of Connecticut. He is also a licensed athletic trainer.

Shuang Yin is a graduate student in the Department of Statistics at the University of Connecticut.

Timothy E. Moore, PhD, is a faculty member for the Statistical Consulting Services, Center for Open Research Resources \& Equipment at the University of Connecticut.

Yuki Murata is a graduate student in the graduate school of education and human development at Nagoya University in Japan.

Johna K. Register-Mihalik, PhD, ATC, is an associate professor in the Department of Exercise and Sport Science at the University of North Carolina Chapel Hill. She is also a licensed athletic trainer.

Lindsay J. DiStefano, PhD, ATC, is an associate professor and department head of the Department of Kinesiology at the University of Connecticut. She is also a licensed athletic trainer.

\section{Acknowledgements}

This study was partially funded by the NATA Research \& Education Foundation.

\section{Authors}

Samantha E. Scarneo-Miller, Douglas J. Casa, Shuang Yin, Timothy E. Moore, Yuki Murata, Johna Kay Register-Mihalik, and Lindsay J. DiStefano 


\title{
IIIAHSP \\ The Internet Joumnal of Allied Health Sciences and Practice \\ Dedicated to allied health professional practice and education \\ Vol. 19 No. 2 ISSN 1540-580X
}

\section{The Precaution Adoption Process Model in Describing Emergency Action Plan Adoption}

\author{
Samantha E. Scarneo-Miller ${ }^{1}$ \\ Douglas J. Casa² \\ Shuang Yin² \\ Timothy E. Moore ${ }^{2}$ \\ Yuki Murata ${ }^{3}$ \\ Johna Kay Register-Mihalik ${ }^{4}$ \\ Lindsay J. DiStefano ${ }^{2}$
}

1. West Virgina University

2. University of Connecticut

3. Nagoya University

4. University of North Carolina at Chapel Hill

United States and Japan

\begin{abstract}
Purpose: The purpose of this study was to identify the ability of the Precaution Adoption Process Model (PAPM) to classify athletic trainers (ATs) and athletic directors (ADs) readiness to act with regards to adopting an emergency action plan (EAP), and describe factors influencing PAPM stages. Method: We used a cross-sectional questionnaire design to evaluate readiness to act for developing an EAP among ATs and ADs working in United States high schools. The PAPM is a participant-driven model to identify someone's readiness to act or change and has seven stages: Unaware, Unengaged, Undecided, Decided not to Act, Decided to Act, Acting and Maintaining. Prevalence ratios (PRs) with $95 \%$ confidence intervals $(95 \% \mathrm{Cl})$ were calculated. Results: A majority of ATs and ADs reported maintaining an EAP in their secondary school (AT "Maintaining" $=84.4 \%, A D$ "Maintaining" $=68.7 \%$ ). ADs were more likely to be staged as "Decided not to Act" (PR=0.14, 95\% $\mathrm{Cl}=0.05,0.41)$ for the development of an EAP compared to ATs. ATs were more likely to be staged as "Maintaining" (PR=1.23, 95\% $\mathrm{Cl}=1.16,1.30)$ for the development of an EAP compared to ADs. Conclusions: The PAPM appears to be able to classify AT and AD readiness to act with adoption of an EAP. However, statistical modeling struggles to identify predictors for the various stages. Future research should aim to use the PAPM stages in the development of tailored interventions.
\end{abstract}

Keywords: health behavior, best practices, policies and procedures, emergency care 


\section{INTRODUCTION}

Sport participation provides undeniable health benefits for our youth, but the evidence-based tactics known to reduce the inherent risks, including the implementation of policies, have not been widely adopted. ${ }^{1-4}$ Emergency action plans (EAPs) allow for a thoroughly thought out, specific plan to be in place to prevent critical delays from occurring. ${ }^{.}$These plans include clear guidelines for all potential personnel involved. Athletic trainers are properly trained in the management of emergency conditions in athletics and should be on the forefront of creating the EAP. Athletic directors, otherwise known as athletic administrators, are in a unique position to influence policy adoption for the entire athletics program. ${ }^{1,2}$ Despite being trained and uniquely positioned to develop and implement EAPs, recent research suggests that not all athletic trainers and athletic directors report adopting an EAP. ${ }^{1,2}$ Specifically, nearly $90 \%$ of athletic trainers (ATs) and $75 \%$ of athletic directors (ADs) report adopting an EAP in secondary school athletics. ${ }^{1,2}$ However, very few of these individuals implement all 12 components cited in the National Athletic Trainers Association's position statement on emergency planning in athletics. Lack of adoption of these no-cost policies leaves secondary school athletics programs ill prepared to properly manage catastrophic injuries. As such, there is a desperate need to identify the behaviors influencing ATs and ADs perceptions for EAP adoption to aid in the development of evidence-based strategies to improve adoption and ensure athlete safety.

The socioecological framework, which includes considerations at the policy, intrapersonal, interpersonal, organizational, and environmental levels, helps conceptualize the various stakeholders that should be involved with the adoption of policies and procedures, such as EAPs. ${ }^{6}$ The policy level influences all of the other levels (intrapersonal, interpersonal, organizational, environmental) as it dictates standard operating procedures for all stakeholders to abide by. EAPs are considered a standard operating procedure as they outline the plan of care should an individual be critically injured requiring emergency care. At the interpersonal level, ATs play a vital role in the direct care of athletes given their extensive educational background in the prevention, recognition, and care of athletic-related injuries. Specifically, ATs are trained in identifying risk hazards and developing EAPs to reduce or address the hazard. At the organizational level, ADs influence decisions of the organization, or in this case, the high school. ADs are often the liaison between operations occurring during the school day and those which happen when school is out, during athletics. While the ATs are the healthcare professionals trained to develop the EAPs, the ADs are the administrators with influence to increase community adoption (e.g., coaches, other administrators, athletes) of the EAP. Collectively, athletic trainers and athletic directors have been identified as the responsible party for the initiation, development and implementation of the EAP. 1,2 Given the role ATs and ADs have in the adoption of EAPs, understanding the similarities and differences in behaviors related to EAP adoption can aid in the understanding of each role within the socioecological framework.

Community-based strategies (e.g., intrapersonal and organizational levels of the socioecological framework) grounded on health behavior improve policy implementation. ${ }^{7-11}$ Health behavior change theories, such as the Transtheoretical Model of Change and the Precaution Adoption Process Model (PAPM), suggest that preventive measures are adopted through a series of decisional changes. ${ }^{6}$ These change models demonstrate success in describing readiness to change in populations with various health conditions/concerns, including osteoporosis, smoking, and mouthguard use..$^{7-12}$ When interventions are designed and tailored to the individual's current stage of behavior, the likelihood for successful change, and ultimately, adoption increases. ${ }^{13}$

The PAPM was first described by Weinstein and Sandman to explain actions regarding health threats in seven different stages (Figure 1)..$^{14}$ The seven stages of the PAPM suggest individuals behave differently depending on the stage they are currently in, and interventions which address these different behaviors facilitate change. For example, interventions for those in the "Unaware" stage may differ vastly from those in the "Acting" phase. The PAPM has been applied to various domains, such as home radon testing, osteoporosis and disaster preparedness, but has yet to be applied in a sports medicine context. ${ }^{7-11}$

The stages of the PAPM may be able to differentiate schools' readiness to act on policy adoption, specifically at the intrapersonal and organizational levels of the socioecological framework. This identification may facilitate the delivery of feedback or resources to promote sustainable policy adoption. Therefore, the primary purpose of this study was to identify the ability of the PAPM to classify $A T$ and $A D$ readiness to act with regards to adopting an EAP, and describe factors influencing PAPM stages. As both ATs and $A D s$ are reported as the primary responsible parties for the initiation, development and implementation of EAPs, it is imperative to begin to understand if these two stakeholder groups have similar health behaviors related to EAP adoption. Therefore, a secondary purpose of this project was to understand health behavior differences between ATs and ADs for EAP adoption.

\section{Hypotheses}

Hypothesis 1. The PAPM will identify a majority of ATs report "Acting" or "Maintaining" with regards to EAP adoption. Further, we hypothesize that older ATs will be more likely to report "Decided not to Act" compared to "Unaware". 
Hypothesis 2. The PAPM will identify a majority of ADs report "Acting" or "Maintaining" with regards to EAP adoption. Further, we hypothesize that access to an AT will result in higher "Acting" and "Maintaining" proportions compared to those who do not have access to an AT.

Hypothesis 3. ATs will have a larger proportion reporting "Acting" and "Maintaining" for EAP adoption, compared to ADs.

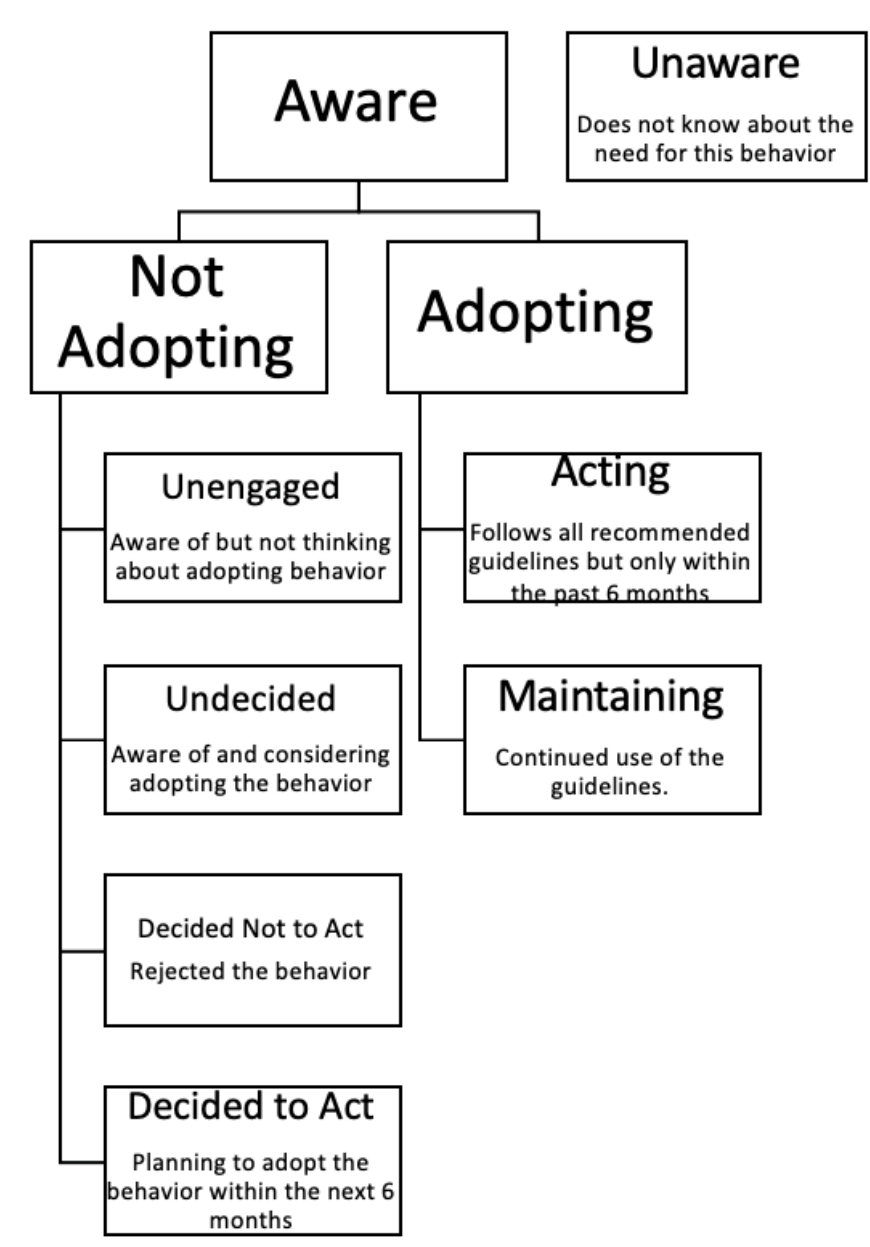

Figure 1. PAPM Stages. Unaware was used as the reference category for the multinomial regression.

\section{METHODS}

We utilized a cross-sectional questionnaire design in this investigation. The questionnaire for this study was created electronically via a platform, Qualtrics LLC (Parvo, UT). The questionnaire asked several questions related to overall EAP policy adoption, and the different suggested components that should be included in an EAP, as outlined in the NATA Position Statement: Emergency Planning in Athletics. ${ }^{5}$ These questions allow for answers to be provided on health behavior stages using the PAPM: Unaware, Unengaged, Undecided, Decided Not to Act, Decided to Act, Acting, Maintaining (Figure 1). Additional questions regarding social determinants (or demographic information), such as age, sex, years in professional role, number of students enrolled in the high school, and highest level of education. The questionnaire is provided in the Appendices.

Surveys were classified as "complete" if $80 \%$ or more of the questions were completed, and an answer to the question "Does your athletics department have a written EAP for managing serious and/or potentially life-threatening sport-related injuries?" was provided. Skip logic was used so that participants were not asked questions about specific components of an EAP if they indicated they did not have an EAP policy at all. Therefore, a total of 980 AT participants and 480 AD participants remained in the analyses for the specific questions related to EAP components. 


\section{Participants \\ Athletic Trainers}

Participants from both the National Athletic Trainers' Association and the Athletic Training Locations and Services (ATLAS) Project distribution list that allowed for email contact for research purposes were contacted $(n=9,642) .{ }^{15}$ Non-respondents received one email follow-up reminder two weeks after the initial request was sent. Of the 9,642 that were invited to take the survey, 1445 surveys were started with 1,104 participants completing the survey, yielding a $11.4 \%$ response rate (female $=56.9 \%$; age $=37 \pm 11$, students enrolled in high school $=1240 \pm 850 ; 15+$ years working in their professional role $=39.5 \%$ ).

\section{Athletic Directors}

Athletic director's (ADs) email addresses were identified through publicly available websites, such as state high school athletics association websites. Email invitations were sent to 9,687 secondary school ADs inviting them to complete the same web-based questionnaire. Two follow-ups were sent to ADs (compared to one with ATs) due to an initial low response rate by ADs. The two follow-up invitations to ADs were sent one and three weeks after the initial distribution. Of the 9,687 emails to potential subjects that were invited to take the survey, 829 surveys were started with 630 completing the survey, yielding a $6.7 \%$ response rate (male $=81.9 \%$; age $=47 \pm 8$, students enrolled in high school $=893 \pm 746 ; 15+$ years working in their professional role $=37.4 \%$; access to an athletic trainer $=73.0 \%$ ).

\section{Statistical Analyses}

The analyses were planned to address the primary purpose of this study, which was to describe the current health behavior of ATs and ADs as it relates to the adoption of an EAP.

Hypothesis 1. Responses in each PAPM stage within the AT responses were summarized and reported as a proportion within each stage. Secondarily, we aimed to examine effects of AT characteristics (age, gender, years in professional role) on the initial EAP question "The athletics department has a written EAP for managing serious and/or potentially life-threatening sport-related injuries". A-priori power analysis using an ordinal logistic regression comparing two groups with an odds ratio (OR) of 1.5 , fraction of 0.7 , cell probabilities with a negative skewness, alpha level of 0.05 and a desired power of $80 \%$ to observe a moderate effect size provided a proposed sample size of 710 subjects. $8,14,16$ We were able to obtain 980 AT responses, indicating power would be reached to observe moderate effect sizes on the PAPM stages.

A multinomial regression was run to compare the different levels of the PAPM on the covariates. We selected 5 AT characteristics as covariates for the multinomial regression model: Age, Students enrolled, Sex, Years in their role at the HS, Years in role in their professional role overall. More information on covariate selection and pairwise relationships can be found in the Supplemental Digital Content. Because of sample size imbalances among levels of categorical AT demographic characteristics, these variables were regrouped. For instance, "years in role at high school" along with "years in professional role" were re-classified into 0-10 years vs $11+$ years (i.e., binary variables). All incomplete cases (missing data) were excluded prior to fitting the model.

Models were fit using the "multinom" function from the nnet package ( $v 3.6 .0$, R Core Team, 2020). ${ }^{17,18}$ To construct the multinomial logit model, one outcome level is chosen as a "reference" level, with the coefficients of other outcomes separately regressed against the reference outcome. In this paper, all regression coefficients are evaluated relative to the "Unaware" PAPM response. Significance of associations between PAPM stages and AT demographic characteristics was evaluated by checking whether the $95 \%$ confidence intervals of ORs for predictors included 1 (where an OR of 1 indicates no difference between stages). Overall model goodness of fit was evaluated using McFadden's R2, where values close to 1 indicate a wellfitting model and values close to zero indicate poor model fit. ${ }^{19}$

Hypothesis 2. AD data was summarized by PAPM stage and reported as a proportion. The questionnaire asked ADs to report if they had access to an AT (yes/no). To compare PAPM stage responses between ADs with access to an AT compared to without access to an AT, prevalence ratios (PRs) were calculated. A PR with a 95\% confidence interval (Cl) excluding 1.00 was considered statistically significant.

Hypothesis 3. The initial analysis provided descriptive data for the current PAPM stage for each respondent and analyzed the similarities or differences between groups (ATs v ADs). As such, our main outcome was the PAPM stage and the main exposure was group. To compare PAPM stage responses between ATs and ADs, PRs were calculated between group responses within each stage. A PR with a $95 \%$ confidence interval $(\mathrm{Cl})$ excluding 1.00 was considered statistically significant. 


\section{RESULTS}

\section{Hypothesis 1: Athletic Trainer PAPM responses}

A majority of athletic trainers reported maintaining an EAP in their secondary school (AT "Maintaining" $=84.4 \%$ ) (Table 1). AT characteristics had very little ability to explain PAPM responses, with the multinomial model having low explanatory power (Rsquared $=0.058$; Table 2). No demographic covariates had significant associations with PAPM stages, regardless of the stages being compared to the reference level ("Unaware"), with all coefficients having confidence intervals including 1 (Table 2).

Table 1. Athletic Emergency Action Plan Adoption. Values are presented as $n(\%)$.

\begin{tabular}{|c|c|c|c|c|c|c|c|c|}
\hline PAPM Stages & & Unaware & Unengaged & Undecided & $\begin{array}{l}\text { Decided not } \\
\text { to act }\end{array}$ & $\begin{array}{c}\text { Decided to } \\
\text { Act }\end{array}$ & Acting & Maintaining \\
\hline \multicolumn{9}{|c|}{ Emergency Action Plan Adoption } \\
\hline $\begin{array}{l}\text { Q1. The athletic } \\
\text { department has a } \\
\text { written emergency } \\
\text { action plan for } \\
\text { managing serious } \\
\text { and/or potentially life- } \\
\text { threatening sport- } \\
\text { related injuries }\end{array}$ & $\begin{array}{l}\text { AT }(n=1104) \\
\text { AD overall } \\
(n=651) \\
\text { Prevalence } \\
\text { ratio }(95 \% \mathrm{Cl})\end{array}$ & $\begin{array}{l}19(1.7) \\
12(1.8) \\
0.93(0.46, \\
1.91)\end{array}$ & $\begin{array}{l}12(1.1) \\
19(2.9) \\
0.37(0.18, \\
0.76)^{*}\end{array}$ & $\begin{array}{l}35(3.2) \\
66(10.1) \\
0.31(0.21, \\
0.47)^{*}\end{array}$ & $\begin{array}{l}4(0.4) \\
17(2.6) \\
0.14(0.05, \\
0.41)^{*}\end{array}$ & $\begin{array}{l}57(8.8) \\
0.56(0.39 \\
0.80)^{*}\end{array}$ & $\begin{array}{l}33(5.1) \\
0.86 \\
(0.56 \\
1.32) \\
\end{array}$ & $\begin{array}{l}447(68.7) \\
1.23(1.16, \\
1.30)^{*}\end{array}$ \\
\hline \multicolumn{9}{|c|}{ If answered "Acting" or "Maintaining" to the question above, respondents were shown the following questions: } \\
\hline $\begin{array}{l}\text { Q2. Is developed and } \\
\text { coordinated with local } \\
\text { EMS, school public } \\
\text { safety officials, on } \\
\text { site medical } \\
\text { personnel or school } \\
\text { medical staff, and } \\
\text { school administrators }\end{array}$ & $\begin{array}{l}\text { AT }(n=976) \\
\text { AD overall } \\
(n=477) \\
\text { Prevalence } \\
\text { ratio }(95 \% C l)\end{array}$ & $\begin{array}{l}22(2.3) \\
16(3.4)\end{array}$ & $\begin{array}{l}0.92(0.41 \\
2.06)\end{array}$ & $\begin{array}{l}1.49(0.93 \\
2.38)\end{array}$ & $\begin{array}{l}6(0.9) \\
1.79(0.73 \\
4.39)\end{array}$ & $\begin{array}{l}20(4.2) \\
1.59(0.97 \\
2.59)\end{array}$ & $\begin{array}{l}60(6.1) \\
30(6.3) \\
0.98 \\
(0.64 \\
1.49)\end{array}$ & $\begin{array}{l}374(78.4) \\
0.94(0.89, \\
1.00)\end{array}$ \\
\hline $\begin{array}{l}\text { Q3. Is distributed and } \\
\text { reviewed by all } \\
\text { relevant athletics staff } \\
\text { members annually }\end{array}$ & $\begin{array}{l}\text { AT }(n=977) \\
\text { AD overall } \\
(n=472) \\
\\
\text { Prevalence } \\
\text { ratio }(95 \% \mathrm{Cl})\end{array}$ & $\begin{array}{l}15(3.2) \\
1.32,(0.74 \\
2.36)\end{array}$ & $\begin{array}{l}6(1.3) \\
1.21(0.47 \\
3.09)\end{array}$ & $\begin{array}{l}23(4.9) \\
1.03(0.63 \\
1.67)\end{array}$ & $\begin{array}{l}8(1.7) \\
0.66(0.27 \\
1.64)\end{array}$ & $\begin{array}{l}25(5.2) \\
1.18(0.75 \\
1.85)\end{array}$ & $\begin{array}{l}38(8.1) \\
1.00 \\
(0.69 \\
1.46) \\
\end{array}$ & $\begin{array}{l}357(75.6) \\
0.98(0.92 \\
1.04)\end{array}$ \\
\hline $\begin{array}{l}\text { Q4. Is rehearsed } \\
\text { annually by } A T, A D \text {, } \\
\text { coaches, other } \\
\text { pertinent medical } \\
\text { personnel }\end{array}$ & $\begin{array}{l}\text { AT }(\mathrm{n}=977) \\
\text { AD overall } \\
(\mathrm{n}=470) \\
\\
\text { Prevalence } \\
\text { ratio }(95 \% \mathrm{Cl})\end{array}$ & $\begin{array}{l}12(2.6) \\
1.52(0.80 \\
2.89)\end{array}$ & $\begin{array}{l}0.56(0.36 \\
0.87)^{*}\end{array}$ & $\begin{array}{l}0.81(0.63 \\
1.03)\end{array}$ & $\begin{array}{l}0.62(0.43 \\
0.89)^{*}\end{array}$ & $\begin{array}{l}50(10.6) \\
1.49(1.11 \\
2.01)^{*}\end{array}$ & $\begin{array}{l}31(6.6) \\
\\
1.07 \\
(0.71 \\
1.61)\end{array}$ & $\begin{array}{l}1.08(0.96 \\
1.21)\end{array}$ \\
\hline $\begin{array}{l}\text { Q5. Is updated } \\
\text { annually by all } \\
\text { relevant athletics staff } \\
\text { members }\end{array}$ & $\begin{array}{l}\text { AT }(n=976) \\
\text { AD overall } \\
(n=470) \\
\text { Prevalence } \\
\text { ratio }(95 \% \mathrm{Cl})\end{array}$ & $\begin{array}{l}16(3.4) \\
0.84(0.46 \\
1.54)\end{array}$ & $\begin{array}{l}11(2.3) \\
1.14(0.57 \\
2.28)\end{array}$ & $\begin{array}{l}33(7.0) \\
0.76(0.50 \\
1.16)\end{array}$ & $\begin{array}{l}12(2.6) \\
0.56(0.26 \\
1.21)\end{array}$ & $\begin{array}{l}23(4.9) \\
1.24(0.77 \\
1.97)\end{array}$ & $\begin{array}{l}36(7.7) \\
0.79 \\
(0.53 \\
1.18)\end{array}$ & $\begin{array}{l}1.05(0.98 \\
1.12)\end{array}$ \\
\hline
\end{tabular}




\begin{tabular}{|c|c|c|c|c|c|c|c|c|}
\hline \multirow{3}{*}{$\begin{array}{l}\text { Q6. Identifies } \\
\text { personnel and their } \\
\text { responsibilities to } \\
\text { carry out the plan of } \\
\text { action with } \\
\text { designated chain of } \\
\text { command }\end{array}$} & AT $(n=977)$ & $10(1.0)$ & $16(1.6)$ & $14(1.4)$ & $9(0.9)$ & $29(3.0)$ & $56(5.7)$ & 843 (86.3) \\
\hline & $\begin{array}{l}\text { AD overall } \\
(n=477)\end{array}$ & $6(1.3)$ & $4(0.8)$ & $20(4.2)$ & $5(1.0)$ & $16(3.4)$ & $43(9.0)$ & $383(80.3)$ \\
\hline & $\begin{array}{l}\text { Prevalence } \\
\text { ratio }(95 \% \mathrm{Cl})\end{array}$ & $\begin{array}{l}0.81 \\
2.23)\end{array}$ & $\begin{array}{l}1.95(0.66 \\
5.81)\end{array}$ & $\begin{array}{l}0.34(0.17 \\
0.67)^{\star}\end{array}$ & $\begin{array}{l}0.88(0.30 \\
2.61)\end{array}$ & $\begin{array}{l}0.88(0.49 \\
1.61)\end{array}$ & \begin{tabular}{|l}
0.64 \\
$(0.43$ \\
$0.93)^{*}$
\end{tabular} & $\begin{array}{l}1.07(1.02 \\
1.13)^{\star}\end{array}$ \\
\hline \multirow{3}{*}{$\begin{array}{l}\text { Q7. Identifies location } \\
\text { of on-site emergency } \\
\text { equipment }\end{array}$} & AT $(n=970)$ & $11(1.1)$ & $8(0.8)$ & $14(1.4)$ & $4(0.4)$ & $22(2.3)$ & $43(4.4)$ & 868 (89.5) \\
\hline & $\begin{array}{l}\text { AD overall } \\
(n=477)\end{array}$ & $2(0.4)$ & $3(0.4)$ & $8(1.7)$ & $1(0.2)$ & $8(1.7)$ & $37(7.8)$ & 419 (87.8) \\
\hline & $\begin{array}{l}\text { Prevalence } \\
\text { ratio }(95 \% \mathrm{Cl})\end{array}$ & $\begin{array}{l}2.70(0.06 \\
12.15)\end{array}$ & $\begin{array}{l}1.31(0.35 \\
4.92)\end{array}$ & $\begin{array}{l}0.86(0.36 \\
2.04)\end{array}$ & $\begin{array}{l}1.97(0.22 \\
17.55)\end{array}$ & $\begin{array}{l}1.35(0.61 \\
3.01)\end{array}$ & $\begin{array}{l}0.57 \\
(0.37 \\
0.87)^{\star}\end{array}$ & $\begin{array}{l}1.02(0.98, \\
1.06)\end{array}$ \\
\hline \multirow{3}{*}{$\begin{array}{l}\text { Q8. Lists contact } \\
\text { information for EMS } \\
\text { and other key } \\
\text { personnel, as well as } \\
\text { facility address, } \\
\text { location }\end{array}$} & AT $(n=970)$ & $11(1.1)$ & $12(1.2)$ & $19(2.0)$ & $5(0.5)$ & $27(2.8)$ & $38(3.9)$ & 858 (88.5) \\
\hline & $\begin{array}{l}\text { AD overall } \\
(n=476)\end{array}$ & $10(2.1)$ & $3(0.6)$ & $16(3.4)$ & $2(0.4)$ & $11(2.3)$ & $40(8.4)$ & 394 (82.8) \\
\hline & $\begin{array}{l}\text { Prevalence } \\
\text { ratio }(95 \% \mathrm{Cl})\end{array}$ & $\begin{array}{l}0.54(0.23 \\
1.26)\end{array}$ & $\begin{array}{l}1.96(0.56 \\
6.92)\end{array}$ & $\begin{array}{l}0.55(0.28 \\
1.07)\end{array}$ & $\begin{array}{l}1.23(0.24 \\
6.30)\end{array}$ & $\begin{array}{l}1.20(0.60 \\
2.41)\end{array}$ & $\begin{array}{l}0.47 \\
(0.30 \\
0.72)^{*}\end{array}$ & $\begin{array}{l}1.07(1.02 \\
1.12)^{*}\end{array}$ \\
\hline \multirow{3}{*}{$\begin{array}{l}\text { Q9. Provides } \\
\text { recommendations for } \\
\text { documentation that } \\
\text { should be taken after } \\
\text { a catastrophic } \\
\text { incident }\end{array}$} & AT (n=967) & $71(7.3)$ & $63(6.5)$ & $130(13.4)$ & $26(2.7)$ & $72(7.4)$ & $46(4.8)$ & $559(57.8)$ \\
\hline & $\begin{array}{l}A D \text { overall } \\
(n=475)\end{array}$ & $32(6.7)$ & $11(2.3)$ & $36(7.6)$ & $17(3.6)$ & $19(4.0)$ & $37(7.8)$ & $323(68.0)$ \\
\hline & $\begin{array}{l}\text { Prevalence } \\
\text { ratio }(95 \% \mathrm{Cl})\end{array}$ & $\begin{array}{l}1.09(0.73 \\
1.63)\end{array}$ & $\begin{array}{l}2.81(1.50 \\
5.29)\end{array}$ & $\begin{array}{l}5.81(3.17 \\
10.64)^{\star}\end{array}$ & $\begin{array}{l}0.75(0.41 \\
1.37)\end{array}$ & $\begin{array}{l}1.86(1.14 \\
3.05)^{\star}\end{array}$ & $\begin{array}{l}0.61 \\
(0.40 \\
0.93)^{*}\end{array}$ & $\begin{array}{l}0.85(0.78 \\
1.92)^{\star}\end{array}$ \\
\hline \multirow{3}{*}{$\begin{array}{l}\text { Q10. Includes } \\
\text { information for } \\
\text { healthcare } \\
\text { professionals } \\
\text { providing medical } \\
\text { coverage which is } \\
\text { included in the review } \\
\text { and rehearsal }\end{array}$} & AT $(n=970)$ & $49(5.1)$ & $44(4.5)$ & $82(8.5)$ & $23(2.4)$ & $55(5.7)$ & $52(5.4)$ & 665 (68.6) \\
\hline & $\begin{array}{l}A D \text { overall } \\
(n=475)\end{array}$ & $40(8.4)$ & $33(6.9)$ & $52(10.9)$ & $27(5.7)$ & $20(4.2)$ & $28(5.9)$ & 275 (57.9) \\
\hline & $\begin{array}{l}\text { Prevalence } \\
\text { ratio }(95 \% \mathrm{Cl})\end{array}$ & $\begin{array}{l}0.60(0.40 \\
0.90)^{\star}\end{array}$ & $\begin{array}{l}0.65(0.42 \\
1.01)\end{array}$ & $\begin{array}{l}0.77(0.56 \\
1.07)\end{array}$ & $\begin{array}{l}0.42(0.24 \\
0.72)\end{array}$ & $\begin{array}{l}1.35(0.82 \\
2.22)\end{array}$ & \begin{tabular}{|l|}
0.91 \\
$(0.58$ \\
$1.42)$ \\
\end{tabular} & $\begin{array}{l}1.18(1.08 \\
1.29)^{*}\end{array}$ \\
\hline \multirow{3}{*}{$\begin{array}{l}\text { Q11. Each venue } \\
\text { (practice field, } \\
\text { competition field, } \\
\text { gym, weight room, } \\
\text { etc.) our athletes play } \\
\text { at has its own EAP }\end{array}$} & AT $(n=977)$ & 38 (3.9) & $41(4.2)$ & $139(14.2)$ & $59(6.0)$ & $155(15.9)$ & $69(7.1)$ & 476 (48.7) \\
\hline & $\begin{array}{l}\text { AD overall } \\
(n=470)\end{array}$ & $12(2.6)$ & $35(7.4)$ & $83(17.7)$ & $46(9.8)$ & $50(10.6)$ & $31(6.6)$ & $213(45.3)$ \\
\hline & $\begin{array}{l}\text { Prevalence } \\
\text { ratio }(95 \% \mathrm{Cl})\end{array}$ & $\begin{array}{l}1.52(0.80 \\
2.89)\end{array}$ & $\begin{array}{l}0.56(0.36 \\
0.87)^{\star}\end{array}$ & $\begin{array}{l}0.81(0.63 \\
1.03)\end{array}$ & $\begin{array}{l}0.61(0.43, \\
0.89)^{*}\end{array}$ & $\begin{array}{l}1.49(1.11 \\
2.01)^{*}\end{array}$ & $\begin{array}{l}1.07 \\
(0.71 \\
1.61) \\
\end{array}$ & $\begin{array}{l}1.08,(0.96, \\
1.21)\end{array}$ \\
\hline
\end{tabular}




\begin{tabular}{|l|l|l|l|l|l|l|l|l|}
\hline $\begin{array}{l}\text { Q12. The EAP is } \\
\text { posted at every } \\
\text { venue }\end{array}$ & $\begin{array}{l}\text { AD overall }(n=976) \\
(n=470)\end{array}$ & $28(2.9)$ & $26(2.7)$ & $52(5.3)$ & $14(1.4)$ & $59(6.0)$ & $59(6.0)$ & $738(75.6)$ \\
& $\begin{array}{l}16(3.4) \\
\text { Prevalence } \\
\text { ratio (95\% Cl) }\end{array}$ & $\begin{array}{l}0.84(0.46, \\
1.54)\end{array}$ & $\begin{array}{l}1.14(0.57, \\
2.28)\end{array}$ & $\begin{array}{l}0.76(0.50, \\
1.16)\end{array}$ & $\begin{array}{l}0.56(0.26, \\
1.21)\end{array}$ & $\begin{array}{l}1.24(0.77, \\
1.97)\end{array}$ & $\begin{array}{l}0.79 \\
(0.53, \\
1.18)\end{array}$ & $\begin{array}{l}1.05(0.98, \\
1.12)\end{array}$ \\
\hline
\end{tabular}

Table 2. Multinomial regression with reference level as Stage 1 "I don't know".

\begin{tabular}{|c|c|c|c|c|c|c|}
\hline \multicolumn{7}{|c|}{$\begin{array}{l}\text { Values Presented as: Odds Ratios (95\% Cl) } \\
\text { P-value }\end{array}$} \\
\hline Observations & 1036 & $\mathrm{R}^{2}$ & 0.058 & & & \\
\hline $\begin{array}{l}\text { PAPM } \\
\text { Stage/Predictors }\end{array}$ & Intercept & Age & $\begin{array}{l}\text { Students } \\
\text { Enrolled }\end{array}$ & Sex & $\begin{array}{l}\text { Years in Role at } \\
\text { High School }\end{array}$ & $\begin{array}{l}\text { Years in } \\
\text { Professional Role }\end{array}$ \\
\hline $\begin{array}{l}\text { Decided Not to } \\
\text { Act }\end{array}$ & $\begin{array}{l}0.41 \\
(0.03,5.62) \\
p=0.508\end{array}$ & $\begin{array}{l}0.64 \\
(0.15,2.75) \\
p=0.549\end{array}$ & $\begin{array}{l}0.30 \\
(0.04,2.08) \\
p=0.222\end{array}$ & $\begin{array}{l}0.14 \\
(0.01,1.83) \\
p=0.132\end{array}$ & $\begin{array}{l}1.30 \\
(0.08,20.84) \\
p=0.851\end{array}$ & $\begin{array}{l}0.34 \\
(0.01,11.76) \\
p=0.547\end{array}$ \\
\hline Unengaged & $\begin{array}{l}1.06 \\
(0.18,6.29) \\
p=0.951\end{array}$ & $\begin{array}{l}1.01 \\
(0.38,2.67) . . \\
p=0.991\end{array}$ & $\begin{array}{l}1.62 \\
(0.75,3.49) \\
p=0.218\end{array}$ & $\begin{array}{l}0.44 \\
(0.08,2.34) \\
p=0.338\end{array}$ & $\begin{array}{l}1.42 \\
(0.20,9.94) \\
p=0.722\end{array}$ & $\begin{array}{l}0.62 \\
(0.05,6.97) \\
p=0.696\end{array}$ \\
\hline Undecided & $\begin{array}{l}1.15(0.24,5.51) \\
p=0.864\end{array}$ & $\begin{array}{l}1.0 \\
(0.46,2.19) \\
p=0.999\end{array}$ & $\begin{array}{l}1.39 \\
(0.73,2.63) \\
p=0.317\end{array}$ & $\begin{array}{l}0.39 \\
(0.10,1.45) \\
p=0.161\end{array}$ & $\begin{array}{l}.73 \\
(0.88,25.55) \\
p=0.071\end{array}$ & $\begin{array}{l}1.11 \\
(0.18,7.09) \\
p=0.908\end{array}$ \\
\hline Decided to Act & $\begin{array}{l}2.64 \\
(0.64,10.80) \\
p=0.178\end{array}$ & $\begin{array}{l}0.92 \\
(0.43,1.95) \\
p=0.823\end{array}$ & $\begin{array}{l}0.99 \\
(0.53,187) \\
p=0.985\end{array}$ & $\begin{array}{l}0.86 \\
(0.24,3.04) \\
p=0.816\end{array}$ & $\begin{array}{l}1.46 \\
(0.31,6.86) \\
p=0.633\end{array}$ & $\begin{array}{l}0.87 \\
(0.14,5.41) \\
p=0.883\end{array}$ \\
\hline Acting & $\begin{array}{l}1.63 \\
(0.37,7.14) \\
p=0.514\end{array}$ & $\begin{array}{l}0.92 \\
(0.43,2.01) \\
p=0.841\end{array}$ & $\begin{array}{l}1.02 \\
(0.54,1.94) \\
p=0.944\end{array}$ & $\begin{array}{l}0.98 \\
(0.27,3.58) \\
p=0.979\end{array}$ & $\begin{array}{l}1.09 \\
(0.20,5.95)\end{array}$ & $\begin{array}{l}2.46 \\
(0.36,16.81) \\
p=0.360\end{array}$ \\
\hline Maintaining & $\begin{array}{l}69.84 \\
(20.67,235.94) \\
\mathrm{P}<0.001^{*}\end{array}$ & $\begin{array}{l}0.66 \\
(0.34,1.27) \\
p=0.211\end{array}$ & $\begin{array}{l}1.38 \\
(0.80,2.39) \\
p=0.249\end{array}$ & $\begin{array}{l}0.50 \\
(0.17,1.49) \\
p=0.213\end{array}$ & $\begin{array}{l}1.60 \\
(0.41,6.19) \\
p=0.497\end{array}$ & $\begin{array}{l}0.85 \\
(0.17,4.23) \\
p=0.842\end{array}$ \\
\hline
\end{tabular}

\section{Hypothesis 2: Athletic Director PAPM Responses}

A majority of ADs reported maintaining the development of an EAP at their secondary school (AD "Maintaining" = 68.7\%) (Table 1). ADs who reported having access to an AT were more likely to report "Maintaining" the development of an EAP, compared with those who do not have access to an AT (with=72.85\%, without=57.83\%; $P R=1.26,95 \% \mathrm{Cl}=1.09,1.45$ ) (Table 3 ). Further, ADs without access to athletic training services were more likely to report being "Undecided" compared to athletic trainers with access to an athletic trainer (with $=8.41 \%$, without $=15.66 \% ; \mathrm{PR}=0.54,95 \% \mathrm{Cl}=0.34,0.85$ ).

Table 3. AD with AT services compared with AD without AT services. Values presented as $n(\%)$.

\begin{tabular}{|c|c|c|c|c|c|c|c|c|}
\hline & & Unaware & Unengaged & Undecided & $\begin{array}{l}\text { Decided } \\
\text { not to act }\end{array}$ & $\begin{array}{l}\text { Decided } \\
\text { to Act }\end{array}$ & Acting & Maintaining \\
\hline \multirow{3}{*}{$\begin{array}{l}\text { The athletic } \\
\text { department has } \\
\text { a written } \\
\text { emergency } \\
\text { action plan for } \\
\text { managing } \\
\text { serious and/or } \\
\text { potentially life- } \\
\text { threatening }\end{array}$} & $\begin{array}{l}\text { AD with } \\
\text { AT } \\
(n=464)\end{array}$ & $7(1.51)$ & $10(2.16)$ & $39(8.41)$ & $9(1.94)$ & $36(7.76)$ & $\begin{array}{l}\text { 25(5.3 } \\
\text { 9) }\end{array}$ & $338(72.85)$ \\
\hline & $\begin{array}{l}\text { AD } \\
\text { without } \\
\text { AT } \\
(n=166)\end{array}$ & $4(2.41)$ & $7(4.22)$ & $26(15.66)$ & $7(4.22)$ & $\begin{array}{l}18(10.84 \\
)\end{array}$ & $\begin{array}{l}8(4.82 \\
)\end{array}$ & $96(57.83)$ \\
\hline & $\begin{array}{l}\text { PR }(95 \% \\
\text { Cl) }\end{array}$ & $\begin{array}{l}0.63 \\
(0.19 \\
2.11)\end{array}$ & $\begin{array}{l}0.51(0.20 \\
1.32)\end{array}$ & $\begin{array}{l}0.54(0.34 \\
0.85)^{*}\end{array}$ & $\begin{array}{l}0.46 \\
(0.17 \\
1.22)\end{array}$ & $\begin{array}{l}0.72 \\
(0.42, \\
1.22)\end{array}$ & $\begin{array}{l}1.12 \\
(0.51, \\
2.43)\end{array}$ & $\begin{array}{l}1.26(1.09 \\
1.45)^{*}\end{array}$ \\
\hline
\end{tabular}




\begin{tabular}{|l|l|l|l|l|l|l|l|}
\hline $\begin{array}{l}\text { Sport-related } \\
\text { injuries }\end{array}$ & & & & & & & \\
\hline
\end{tabular}

\section{Hypothesis 3: Comparison between AT and AD}

Of the stages related to not adopting an EAP, $4.9 \%$ of ATs and $8.8 \%$ of ADs reported "Decided to Act" on developing an EAP. Less than $1 \%(0.4 \%)$ of ATs and $2.6 \%$ of ADs reported they "Decided not to Act" with an EAP (Table 1). Athletic directors were more likely to be staged as "Unengaged" ( $P R=0.37,95 \% \mathrm{Cl}=0.18,0.76)$, "Undecided" $(0.31,95 \% \mathrm{Cl}=0.21,0.47)$, "Decided not to Act" (PR=0.14, $95 \% \mathrm{Cl}=0.05,0.41)$, "Decided to Act" (PR= 0.56, 95\% $\mathrm{Cl}=0.39,0.80)$ for the development of an EAP. ATs were more likely to be staged as "Maintaining" ( $P R=1.23,95 \% \mathrm{Cl}=1.16,1.30)$ for the development of an EAP compared to ADs.

\section{DISCUSSION}

Despite the evidence for the need for an EAP, previous research has found the prevalence of schools adopting an EAP is not $100 \%{ }^{1,2}$ While previous studies have aimed at evaluating factors influencing adoption, the decision-making process for the development of an EAP remains unknown..$^{20,21}$ Therefore, we aimed to evaluate the decision-making process for the development of an EAP using the PAPM. Our findings confirm prior literature demonstrating that EAP adoption is not universal and expands the literature by suggesting the use of the PAPM can identify differences in readiness to act between stakeholders. This information can help refine strategies and resources to promote future adoption of EAPs within high school sport.

Understanding factors influencing stakeholder's ability or desire to develop EAPs is essential. The socioecological framework can help us to conceptualize that adoption of EAPs may be multifaceted and involves many different stakeholders, including ATs and ADs. To date, specific factors, such as a state requirement, perceived financial limitations, and stakeholder buy-in, appear to be the most influencing factors for a high school's ability or desire to develop EAPs.20,21 However, these factors may not explain all barriers and facilitators to EAP adoption. The PAPM provides context and an in-depth analysis as to one's readiness to act to adopt these policies. ${ }^{14,16}$ In this study, the PAPM was able to describe the readiness to act of ATs and ADs in the secondary school setting. While it is a positive finding that a majority of ATs and ADs report "Adopting" and "Maintaining" for all components, it is important to identify why this is not $100 \%$. Previous findings on traditional public health topics such as osteoporosis behaviors and colorectal screenings find approximately $16 \%$ of a sample are classified as "Acting" and between $35-45 \%$ as "Maintaining.", 722,23 It is interesting that our findings suggest higher classifications of "Acting" and "Maintaining," but yet we are not seeing that all ATs and $A D s$ are reporting adoption of these lifesaving plans. Regardless, the ability to find that the PAPM is able to assist with the classification of readiness to act for both ATs and ADs is encouraging.

The components of an EAP policy with the most variability in readiness to act for both ATs and ADs were "rehearsed annually by $A T, A D$, coaches, other pertinent medical personnel" and "each venue (practice field, competition field, gym, weight room, etc.) our athletes play at has its own EAP." Rehearsal of the emergency plan allows for all involved stakeholders to be familiar with the procedures to carry out in the event of a catastrophic injury. Failure to adopt a rehearsal strategy will lead to chaos, confusion and, ultimately, a lack of proper care for the patient. Within those not adopting this component, nearly $15 \%$ of ATs and $18 \%$ of ADs report being "Undecided" to adopt this policy. Developing interventions that are tailored to each of the stages within the PAPM may provide a unique strategy to improving EAP adoption. In the example of developing a rehearsal strategy, interventions aimed at addressing the individual's belief about an emergency situation's likelihood of happening may be beneficial. Additionally, those who classify as "Unengaged" from any component may benefit from information about the prevalence of emergency situations in sport - how many exertional heat illnesses occur every year, how many times an athletic trainer calls EMS in the high school setting, out of hospital cardiac arrest survival rates.

Access to AT services appeared to have an effect on the health behavior stages of athletic directors. Overall, when the AD reported access to an AT, they were more likely to be "Maintaining" for a policy on the development of EAPs (72.6\%) than when they did not have access to AT services (57.2\%). We also see proportional differences in ADs report of "Maintaining" for "EAP Is updated annually by all relevant athletics staff members" (AD with access to an $A T=74.8 \%$, without access to an $A T=61.9 \%$ ) and "the EAP is posted at every venue" (AD with access to an $A T=74.8 \%$, without access $(61.9 \%)$. Previous research has provided preliminary evidence to support that access to AT services to be associated with an increase in policy adoption. ${ }^{2}$ As the AT is identified as the primary stakeholder involved in the development of the EAP, this is not a surprising finding. Further, it is possible that the decisionmaking process that the schools, including the AD, navigated through to decide to provide AT services is the same decision-making process when looking at EAP development. In other words, the AD consciously decided to ensure the student-athletes had access to healthcare when playing sports. As such, they have adopted what we may term as a 'health and safety' mindset which may be translating into the EAP findings we report in this study. As not every high school in the United States has access to AT services, there is a need to continue to focus efforts on improving access to athletic training services. 
Findings from our multinomial regression indicate no relationship or association between the PAPM stages of the question "Does the athletic department have a written EAP?" and AT characteristics. In fact, many estimates showed large confidence intervals. Overall, these results suggest the multinomial regression model presented here is unable to identify associations between PAPM responses and AT characteristics. One possible explanation for this is the substantial imbalance present in the response variable, with more than $80 \%$ of respondents selecting the highest PAPM stage (" $84.4 \%$ ", "Maintaining"). Such a strong imbalance can generate biased and misleading results. ${ }^{24,25}$ With imbalanced datasets, the algorithm does not necessarily obtain enough information from the minority categories, causing the prediction to be biased towards the majority categories. Another possible source of bias is from imbalance in the predictor variables, with some categories having relatively few observations. This coupled with the imbalance in the response variables can lead to partial separation, as indicated by large confidence intervals on parameter estimates. ${ }^{26}$ Possible solutions include: using a Bayesian approach to borrow information from the sparse and imbalanced settings or bias reduction models can be fit that make use of the "Poisson trick" to accommodation separation issues. ${ }^{24,25,27}$ Future research may aim to increase the sample size to allow for higher responses in the "not adopting" stages to allow for a better model fit.

An interesting finding from this investigation was that when looking at the "Not Adopting" stages (Unaware, Unengaged, Undecided, Decided not to Act, Decided to Act) within the question "Does the athletic department have a written EAP for managing serious and/or life threatening sport-related injuries?", ADs were significantly more likely to be Unengaged, Undecided, Decided not to Act and Decided to Act, when compared to ATs. Athletic trainers are healthcare professionals trained in the prevention, recognition and treatment of injuries. One of the topics ATs are trained in is healthcare administration and policies and procedures and as such, are the person most often involved in the development of the EAP. ${ }^{28,29}$ Athletic directors, on the other hand, are in charge of the overall athletics program and most often do not have advanced healthcare training. Cooperatively, both the AT and AD have been demonstrated to be involved with the initiation, development and implementation of the EAP.1,2 This may be a contributing factor for why more ATs report Acting and Maintaining of an EAP compared to ADs along with the significant differences across the "Not Adopting" stages.

\section{Limitations \& Future Directions}

Despite being a larger sample size in the context of sports medicine and policy adoption review, the distribution of responses was heavily skewed to two of the PAPM responses (finding more than 70\% of the responses in the "Acting" and "Maintaining" stages), and thus was underpowered to identify differences using the multinomial regression. While this finding is typical in traditional public health literature, we find the stage differentials to be exaggerated further in this sports medicine context. This proves difficult when attempting to run statistical analysis, as the data are not ordinal and the distribution is too imbalanced for a standard multinomial regression. Continued methodological advances are needed to explore the best way to identify the readiness to act of the sportsmedicine community. It is plausible that the survey design itself may have led to these impactful differences. Previous research on the PAPM has identified different interventional strategies for the different stages to aid in the changing of health behavior. Future research should also aim to evaluate the development of tailored strategies using the health behavior stages on adoption.

\section{CONCLUSIONS}

Ultimately, results from this investigation will allow for a more global, comprehensive approach for safety policy implementation to occur. As commonalities are often identified between health behaviors and policy implementation, the results from this study may be able to be utilized when considering increased policy compliance within other domains such as concussion, heat stroke, and musculoskeletal injuries. We are optimistic the results from this investigation will provide a benchmark to provide guidance for implementation procedures for overall sport safety implementation. The utilization of traditional public health models, such as the Precaution Adoption Process Model (PAPM) and the socio-ecological framework provide new and innovative strategies for sports medicine to utilize, especially with developing an understanding of the factors influencing policy adoption. Most importantly, though ATs and ADs report adoption of an EAP, these results support that the EAP may not be comprehensive or inclusive of all recommendations set forth in the best practice documents. ${ }^{1,2}$ Information from the PAPM, such as the finding that ATs were more likely to be staged as "Maintaining" for the development of an EAP compared to ADs, highlights differences in behavior related to EAP adoption across stakeholder groups. Future research may aim to address these differences to gain knowledge and support for EAP adoption across all levels of the socioecological framework. Additionally, future PAPM data related to sports medicine best practice adoption may be able to differentiate schools' readiness to act for each best-practice, providing essential information for where to begin when creating strategies to improve policy adoption. This knowledge allows us to begin to create strategies that are tailored to the current readiness to act with the key stakeholders, an approach that has shown much success in traditional public health intervention research. $7,8,14,16$ 


\section{REFERENCES}

1. Scarneo SE, Distefano LJ, Stearns RL, Register-Mihalik JK, Denegar CR, Casa DJ. Emergency action planning in secondary-school athletics: A comprehensive Evaluation of Current Adoption of Best Practice Standards. Journal of Athletic Training. 2019;54(1):99-105.

2. Scarneo-Miller SE, DiStefano LJ, Register-Mihalik JK, Stearns RL, Denegar CR, Casa DJ. Athletic administrators report of emergency action plan adoption in secondary school athletics. 2019;11(3):1-10. doi.org/10.18666/JASM-2019-V11-I39240

3. Kerr ZY, Register-Mihalik JK, Pryor RR, Hosokawa Y, Scarneo-Miller SE, Casa DJ. Compliance with the National Athletic Trainers' Association Inter-Association task force preseason heat-acclimatization guidelines in high school football. Journal of Athletic Training. 2019;54(7):749-757. doi:10.4085/1062-6050-373-18

4. Kerr ZY, Scarneo-Miller SE, Yeargin SW, et al. Exertional Heat-Stroke Preparedness in High School Football by Region and State Mandate Presence. Journal of Athletic Training. 2019;54(9):921-928. doi:10.4085/1062-6050-581-18

5. Andersen J, Courson RW, Kleiner DM, McLoda TA. National Athletic Trainers' Association Position Statement: Emergency Planning in Athletics. Journal of Athletic Training. 2002;37(1):99-104.

6. Scarneo S, Kerr ZY, Kroshus E, et al. The Socio-Ecological Framework: A Multi-Faceted Approach to Prevent SportRelated Death in High School Sports. Journal of Athletic Training. 2019;54(4):356-360. doi:10.4085/1062-6050-173-18

7. Blalock SJ, DeVellis RF, Giorgino KB, DeVellis BM, Gold DT, Dooley MA, Anderson JJB, Smith SL. Osteoporosis Prevention in Premenopausal Women: Using a Stage Model Approach to Examine the Predictors of Behavior. Health Psychology. 1996;15(2): 84-93.

8. Elliott JO, Seals BF, Jacobson MP. Use of the Precaution Adoption Process Model to examine predictors of osteoprotective behavior in epilepsy. Seizure. 2007;16(5):424-437. doi:10.1016/j.seizure.2007.02.016

9. Everson ES, Taylor AH, Ussher M. Determinants of physical activity promotion by smoking cessation advisors as an aid for quitting: Support for the Transtheoretical Model. Patient Education and Counseling. 2010;78(1):53-56. doi:10.1016/j.pec.2009.05.004

10. Collins JE, Katz JN, Donnell-Fink LA, Martin SD, Losina E. Cumulative Incidence of ACL Reconstruction After ACL Injury in Adults: Role of Age, Sex, and Race. The American Journal of Sports Medicine. 2013;41(3):544-549.

11. Howat P, Jones S, Hall M, Cross D, Stevenson M. The PRECEDE-PROCEED Model: application to planning a child pedestrian injury prevention program. Injury Prevention: Journal of the International Society for Child and Adolescent Injury Prevention. 1997;3(4):282-287.

12. Tatar O, Perez S, Naz A, Shapiro GK, Rosberger Z. Psychosocial correlates of HPV vaccine acceptability in college males: A cross-sectional exploratory study. Papillomavirus Research. 2017;4:99-107. doi:10.1016/j.pvr.2017.11.001

13. Marcus BH, Emmons KM, Simkin-Silverman LR, et al. Evaluation of motivationally tailored vs. standard self-help physical activity interventions at the workplace. American Journal Health Promotion. 1998;12(4):246-253. doi:10.4278/0890-117112.4 .246

14. Weinstein ND, Sandman PM. A Model of the Precaution Adoption Process: Evidence from Home Radon Testing. Health Psychology : Official Journal of the Division of Health Psychology, American Psychological Association. 1992;11(3):170_ 180.

15. Athletic Training Locations and Services (ATLAS Project). Korey Stringer Institute. Accessed February 15, 2018. https://ksi.uconn.edu/nata-atlas/

16. Weinstein ND, Sandman PM, Blalock SJ. The Precaution Adoption Process Model. In: Health Behavior and Health Education. Vol 21. 4th ed. Jossey-Bass; 2008:123-147.

17. Ripley BD, Venables WN. Feed-Forward Neural Networks and Multinomial Log-Linear Models. R Package Version 7.312.; 2016. https://CRAN.R-project.org/package=nnet.

18. Venables WN, Ripley BD. Modern Applied Statistics with S. Fourth. Springer; 2002.

19. McFadden D. Regression-Based Specification Tests for the Multinomial Logit Model. Journal of Econometrics. 1987;34:63-82. doi:10.2307/1910997

20. Johnson S, Norcross MF, Bovbjerg V, Koester M. Barriers and facilitators of implementing emergency action plans in Oregon high schools. Journal of Athletic Training. 2018;53(6(S)):S-216.

21. Scarneo-Miller SE, DiStefano LJ, Singe SM, Register-Mihalik JK, Stearns RL, Casa DJ. Emergency action plans in secondary schools: barriers, facilitators, and social determinants affecting implementation. Journal of Athletic Training. 2020;55(1):80-87. doi:10.4085/1062-6050-484-18

22. Hester CM, Born WK, Yeh HW, Young KL. Decisional stage distribution for colorectal cancer screening among diverse, low-income study participants. Health Education Research. 2015;30(3):400-411. doi:10.1093/her/cyv006

23. Costanza ME, Luckmann R, Stoddard AM, Avrunin JS, White MJ, Stark JR, Clemow L, Rosal MC. Applying a stage model of behavior change to colon cancer screening. Preventive Medicine. 2005;41(3-4):707-719.

doi:10.1016/j.ypmed.2004.12.013 
24. Johndrow JE, Smith A, Pillai N, Dunson DB. MCMC for imbalanced categorical data. Journal of the American Statistical Association. 2019;114(527):1394-1403.

25. Yap BW, Rani KA, Rahman HAA, Dunson DB. An application of oversampling, undersampling, bagging and boosting in handling imbalanced datasets. Presented at the: Proceedings of the First International Conference on Advanced Data and Information Engineering (DaEng-2013); Springer, Singapore.

26. Albert A, Anderson JA. On the existence of maximum likelihood estimates in logistic regression models. Biometrika. 1984;71(1):1-10.

27. Kosmidis I, Firth D. Multinomial Logit Bias Reduction via the Poisson Log-Linear Model. 2011;98(3):755-759.

28. The Commission on Accreditation of Athletic Training Education. Standards for the Accreditation of Professional Athletic Training Programs. Effective July 1, 2012.

29. The Commission on Accreditation of Athletic Training Education. 2020 Standards for Accreditation of Professional Athletic Training Programs. Master's Degree Programs. Effective date: July 1, 2020. 
Figure 1. Precaution Adoption Process Model (PAPM) Stages. Unaware was used as the reference category for the multinomial regression. 